\title{
Alpha-1-antitrypsin genotyping with mouthwash specimens
}

\author{
R.A. Stockley*, E.J. Campbell ${ }^{\#}$
}

\begin{abstract}
Alpha-1-antitrypsin genotyping with mouthwash specimens. R.A. Stockley, E.J. Campbell. (C) ERS Journals Ltd 2001.

ABSTRACT: $\alpha_{1}$-antitrypsin $\left(\alpha_{1}\right.$-AT) deficiency is diagnosed as a two-stage procedure (concentration and phenotype). However the latter does not provide clues to the presence of null genes without family studies and obtaining blood from patients at a distance often proves difficult. The aim of the study was to assess the feasibility of genotyping $\alpha_{1}$-AT using buccal cells.

Mouthwash specimens were sent by 84 patients (with a variety of phenotypes of $\alpha_{1}$-antitrypsin) through the post. Deoxyribonucleic acid (DNA) was isolated from buccal cells in each sample and subjected to polymerase chain reaction (PCR) using a genotyping kit to detect the $S$ and $Z$ alleles.

Eighty-three of 84 samples received were suitable for amplification. The specific primers successfully identified the $S$ and $Z$ alleles in each case. However, five of the 35 samples obtained from patients thought to be $Z$ allele homozygotes were found to be heterozygotes for another severe deficiency allele.

These data confirm the feasibility of "at distance" testing for $\alpha_{1}$-antitrypsin deficiency alleles using buccal cells from mouthwash samples. The results raise the possibility that other deficiency alleles are more common than has previously been suspected. Eur Respir J 2001; 17: 356-359.
\end{abstract}

The detection of $\alpha_{1}$-antitrypsin $\left(\alpha_{1}\right.$-AT) deficiency and its implications for families is not always straightforward. Samples identified as having an $\alpha_{1}$-AT concentration outside the normal range may have a variety of genotypes. These would include the MZ genotype which is probably not a risk factor [1], although some over representation in patients with chronic obstructive pulmonary disease (COPD) has been observed [2], the SZ genotype which also appears to represent little risk to the development of significant COPD [3] and the ZZ genotype which is a well accepted risk factor. In addition, measuring $\alpha_{1}$-AT levels will identify many subjects who carry the null gene which would include genotypes such as the $\mathrm{M}$ null, $\mathrm{S}$ null, $\mathrm{Z}$ null and null null subjects. Whereas the latter two are also recognized risk factors, the $\mathrm{M}$ null and the $\mathrm{S}$ null have received little attention. Finally genetic variants such as the $\mathrm{F}$ phenotype present an increased risk for the development of disease because of its poor function [4] although the concentration is normal.

Phenotyping may help in determining the interpretation of both a normal and low level. However, phenotyping is complex, requiring the preparation of good quality isoelectric focusing gels (fig. 1) and interpretation of the phenotype requires skilled readers. Furthermore, because phenotyping requires the presence of the $\alpha_{1}$-AT protein in the serum, this method fails to identify null genes. Hence it has become convention to refer to many of the phenotypes as Pi Z,
*Dept of Medicine, Queen Elizabeth Hospital, Birmingham, UK and ${ }^{\#}$ Heredilab Inc, University of Utah, Salt Lake City, Utah, USA.

Correspondence: R.A. Stockley, Dept of Medicine, Queen Elizabeth Hospital, Edgbaston, Birmingham, B15 2TH, UK

Fax: 441216978256

Keywords: Alpha-1-antitrypsin deficiency

genotyping

polymerase chain reaction

Received: June 222000

Accepted after revision October 242000

This project was part of the ADAPT programme funded through a noncommercial grant by Bayer Corporation USA. Asta/Zeneca Diagnostics provided reagents.

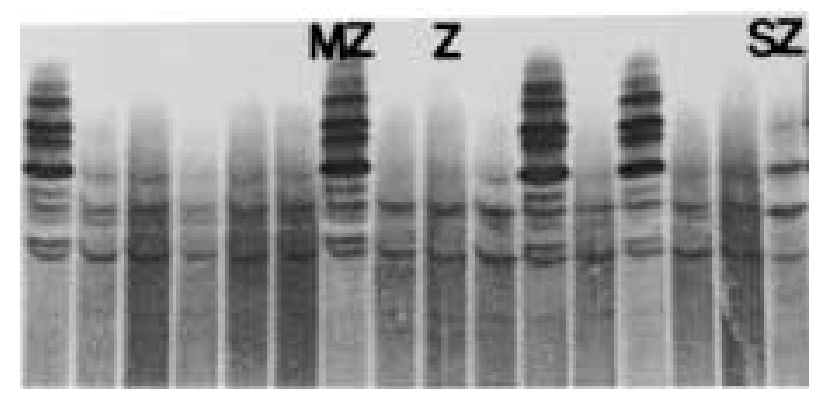

Fig. 1. $-\alpha_{1}$-antitrypsin phenotyping results from finger prick test isoelectric focussing gels indicating patient samples related to typical band patterns for Pi M (the majority) and the Pi MZ, Pi SZ and Pi Z phenotypes as labelled. Note that the band patterns for patients with an MM \& M null or ZZ and Z null genotype would appear identical. 
Pi S, Pi M etc. since the presence of a null gene can only be inferred (at present) by the concentration or the results of family studies.

The alternative approach of genotyping using gene specific primers, is potentially the most accurate. Furthermore, genotyping can easily be carried out using deoxyribonucleic acid (DNA) of any source from the patient and does not therefore specifically require a blood test either by phlebotomy or finger-prick and hence access to medical care. Ideally, and noninvasively, genotyping can be carried out on buccal cells [5], hence providing the opportunity to obtain a simple mouthwash directly from the patient that can be sent through the post.

At present, one such kit is available (the ELUCIGENE AAT from Astra Zeneca Diagnostics, Cheshire, UK) which is able to determine the copy numbers of $\mathrm{S}$ and $\mathrm{Z}$ alleles. The use of this kit has been explored with buccal washes in a variety of patients with a wide spectrum of phenotypes that had been determined previously. The comparison of the genotyping with these phenotypes is reported and its strengths and current weaknesses emphasized.

\section{Methods}

Mouthwash sample collection kits were sent to 100 patients with a variety of known $\alpha_{1}$-AT phenotypes selected to cover a range of common phenotypes but especially those identified as $\mathrm{Pi} \mathrm{Z}$. The kits included a waterproof screwtop bottle, a plastic postal container, a sachet of saline, a padded return postal bag and an instruction leaflet. The patients were asked to fill the bottle to the appropriate level $(10 \mathrm{~mL})$, with Saline then swill this around the mouth for $20 \mathrm{~s}$ and spit it back into the bottle. Samples were then packed and posted to the laboratory.

Preparation of genomic deoxyribonucleic acid from mouthwash samples

The sample was centrifuged at $800 \times g$ for $10 \mathrm{~min}$ at room temperature. The supernatant was carefully removed using a pipette and discarded, whilst the pellet was resuspended by vortex mixing following the addition of $500 \mu \mathrm{L}$ of $10 \mathrm{mM} \mathrm{NaCl}$ (Sigma-Aldrich Company Limited, Poole, Dorset, UK)/10 mM ethylenediamine tetraacetic acid (EDTA) (Sigma) at pH 8.0. The suspension was transferred to a microcentrifuge tube, centrifuged at $12,000 \times g$ for $1 \mathrm{~min}$ at room temperature and the supernatant discarded. Five hundred microlitre of $50 \mathrm{mM} \mathrm{NaOH}$ (Sigma) was then added to each microcentrifuge tube and the pellet resuspended by vortex mixing. The sample was then incubated in a boiling water bath for $5 \mathrm{~min}$ and $100 \mu \mathrm{L}$ of $1 \mathrm{M}$ Tris-base/ $\mathrm{HCl}$ ( $\mathrm{pH}$ 6) (Sigma) was added to the sample. Each sample was then centrifuged for $1 \mathrm{~min}$ at $12,000 \times g$ and the purified DNA was harvested with the supernatant as described [5].

\section{Polymerase chain reaction}

Each sample underwent a PCR [5] involving two

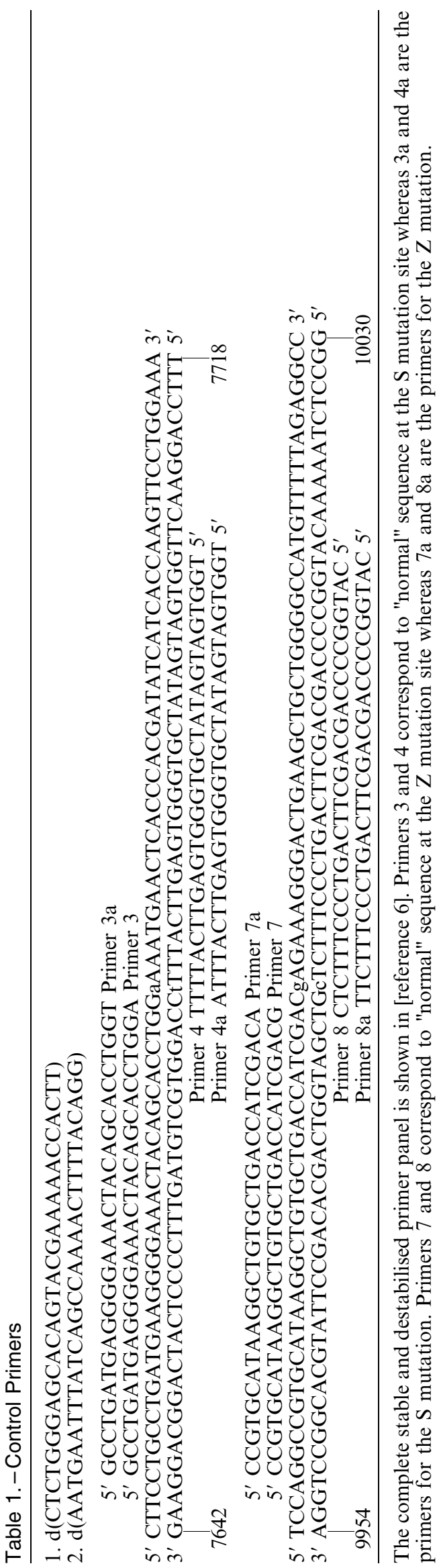


different sets of Amplification Refractory Mulation Systems (ARMS) primers, the first being complementary to the normal sequence at the $\mathrm{Pi} Z$ or the PiS locus (stock primer mix A), the second to the $\mathrm{Z}$ or $\mathrm{S}$ mutant genotype sequence at the $\mathrm{Pi} \mathrm{Z}$ or $\mathrm{Pi} \mathrm{S}$ locus (stock primer mix B), derived from the known sequences. These primers are shown in table 1 and described in detail in reference [6]. Both sets of primers also contained two sets of control primers (regions of the apolipoprotein B and ornithine decarboxylase genes) as proof of amplification. Sixty-four microlitres of sterile distilled $\mathrm{H}_{2} \mathrm{O}$ (Amersham Pharmacia Biotech, Little Chalfont, Bucks, UK) were added to $4 \mu \mathrm{L}$ of Amplitaq Gold (Astra Zeneca Diagnostics) and $4 \mu \mathrm{L}$ of dilution buffer; the resultant mixture was termed Amplitaq Gold diluted enzyme. One hundred and thirty six $\mu \mathrm{L}$ of stock primer mix A or stock primer mix B were then added to $17 \mu \mathrm{L}$ of Amplitaq Gold diluted enzyme in a microcentrifuge tube, the resultant mixtures were carefully mixed using a pipette and were referred to as prepared reaction mix $\mathrm{A}$ or prepared reaction mix $\mathrm{B}$ respectively.

Two and a half microlitre of test DNA sample or positive control DNA sample (purified DNA from PiM individual) were added to each of a pair of microcentrifuge tubes; the first containing $22.5 \mu \mathrm{L}$ of prepared reaction mix $\mathrm{A}$, whilst the second contained $22.5 \mu \mathrm{L}$ of prepared reaction mix $\mathrm{B}$. The negative control consisted of prepared reaction mix A or B only. Finally 1 drop of mineral oil (Sigma) was added to each tube in order to prevent evaporation of the reaction mix during the thermal cycling process.

The amplification procedure consisted of a time delay enzyme activation step of $20 \mathrm{~min}$ at $94^{\circ} \mathrm{C}$, followed by 32 cycles of $2 \mathrm{~min}$ at $94^{\circ} \mathrm{C}$ (denaturation), $1 \mathrm{~min}$ at $62^{\circ} \mathrm{C}$ (annealing) and $1 \mathrm{~min}$ at $72^{\circ} \mathrm{C}$ (extension); followed by a final extension step of $10 \mathrm{~min}$ at $72^{\circ} \mathrm{C}$.

\section{Gel electrophoresis}

Separation and determination of the products of the PCR was achieved by electrophoresis through agarose gel. Each sample pair was run in adjacent lanes, this resulted in a characteristic pattern of bands that was unique to a genotype (Pi M, Pi MZ, Pi MS, Pi SS, Pi SZ or Pi ZZ), thus enabling the genotype of each sample to be determined. Fifteen $\times 12 \mathrm{~cm}$ horizontal submarine gels with $1.5 \mathrm{~mm} \times 5 \mathrm{~mm}$ combs suspended $1 \mathrm{~mm}$ above the gel tray were prepared using $100 \mathrm{~mL}$ of TBE (134 mM tris-base, $74.9 \mathrm{mM}$ boric acid and $2.55 \mathrm{mM}$ EDTA, pH 8) containing 3\% (w/v) NuSieve 3:1 agarose (FMC Bioproducts, Flowgen, Lichfield, Staffordshire, UK) and $0.1 \mathrm{mg} \cdot \mathrm{mL}^{-1}$ ethidium bromide. TBE containing $0.1 \mathrm{mg} \cdot \mathrm{mL}^{-1}$ ethidium bromide (Sigma) was used as the running buffer. Twenty microlitre of PCR product was mixed with $10 \mu \mathrm{L}$ of gel loading buffer (30\% (v/v) glycerol (Sigma) and $0.1 \%$ (w/v) bromophenol blue (Sigma)), and $20 \mu \mathrm{L}$ of this mixture added per lane. The molecular weight marker lane contained $1 \mu \mathrm{g}$ of 50 basepair marker (Amersham Pharmacia) dissolved in $12 \mu \mathrm{L}$ of sterile distilled $\mathrm{H}_{2} \mathrm{O}$ (Amersham Pharmacia) and $8 \mu \mathrm{L}$ of gel loading buffer. Electrophoresis was then carried out at $5 \mathrm{~V} \cdot \mathrm{cm}^{-1}$ until the dye front had migrated $\sim 4 \mathrm{~cm}$ towards the anode. After electrophoresis the gels were placed upon a ultra violet transilluminator at $260 \mathrm{~nm}$ and photographed.

\section{Results}

Of the 100 sample kits sent out, 84 were returned and 83 were suitable for assessment. These included samples from 37 patients with a $\mathrm{Pi} Z$ phenotype, $23 \mathrm{Pi} \mathrm{MZ,}$ six Pi MS, six Pi SZ, five Pi M, two Pi S, one Pi FZ, one Pi M null (M Malton determined previously) and two Pi SX (where $\mathrm{x}$ is a phenotype not characterized but not $\mathrm{M})$.

All samples assessed gave clear bands following PCR and agarose electrophoresis (figure 2) and in 75 cases the genotype was as predicted and concordant with the phenotype including the $\mathrm{Pi} \mathrm{M}$ null patient where the genotype was typical of an MM homozygote (i.e. no S or $\mathrm{Z}$ mutant allele). In addition the two Pi SX and the Pi FZ provided results indicating the presence of only one copy of the $\mathrm{S}$ and $\mathrm{Z}$ gene respectively as would also be predicted.

However, the remaining five samples all identified phenotypically as $\mathrm{Pi} \mathrm{Z}$ appeared genotypically to be MZ heterozygotes i.e. only one copy of the Z allele was detected. Subsequent gene sequence analysis showed three to be Pi ZM malton, one to be Pi ZM procida and one to be Pi ZP lowell.

\section{Discussion}

In clinical practice the diagnosis of $\alpha_{1}$-AT deficiency is often made late in the disease [7]. Once an index case is identified it becomes necessary to screen siblings and other family members mainly to detect other severely deficient subjects, preferably before or in the early stages of development of lung function impairment. This facilitates early intervention aimed at changing

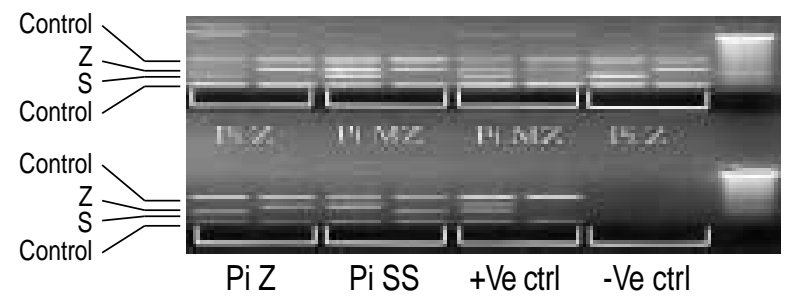

Fig. 2. - Gels of polymerase chain reaction products. The upper and lower bands represent the control primers and the bands in between are the $\alpha_{1}$-antitrypsin specific products. Each sample is run as two tracks. The left hand track between the controls detects the normal sequence around the site of $\mathrm{S}$ mutation (lower) and normal sequence around the $\mathrm{Z}$ mutation site (upper). The right hand track of each pair represents the $S$ mutation sequence (lower) or $\mathrm{Z}$ mutation sequence (upper). For instance $\mathrm{Pi} \mathrm{Z}$ (a ZZ homozygote) detects a normal sequence at the $\mathrm{S}$ mutation but no normal sequence at the $\mathrm{Z}$ mutation alone (left panel) and the $\mathrm{Z}$ mutation sequence (right panel). MZ shows both normal sequences of $\mathrm{Z}$ and $\mathrm{S}$ mutation (left hand) and $\mathrm{Z}$ mutation sequence (right panel). The positive control is an MM homozygote with both bands (normal sequence at $\mathrm{Z} \&$ $\mathrm{S}$ mutation sites) seen in the left hand panel and no $\mathrm{Z}$ or $\mathrm{S}$ mutation sequences identified in the right panel. 
lifestyle mainly with respect to smoking [8] or environmental or occupational pollution. Screening can often be difficult with family members widely scattered and local facilities for testing may be difficult to involve or inappropriate. The current study demonstrated that a simple mouthwash to collect and deliver cellular material by post enables $\alpha_{1}$-AT genotypes to be determined readily.

The current test (ELUCIGENE AAT; Astra Zeneca Diagnostics) provides rapidly and easily readable results and offers a realistic methodology for family and even epidemiological studies for population screening of $\alpha_{1}$-AT and other gene defects or polymorphisms.

The usefulness of such a test depends on its specificity and lack of false negative results. The $\alpha_{1}$-AT protein is highly polymorphic with over 75 phenotypes described, most of which do not influence $\alpha_{1}$-AT concentration or function. However, although the M, S and $\mathrm{Z}$ phenotypes are the most common, a variety of relatively inactive genes have been described including the $\mathrm{F}$ phenotype [4] and I phenotype [9] as well as a series of null genes where little or no product is secreted [10].

Although these variants, especially the null genes, are thought to be rare, their detection in heterozygotes depends on suspicion through family studies. As they do not express or secrete protein they cannot be identified on phenotype analysis and thus Pi Z null or Pi $\mathrm{M}$ null heterozygotes will appear to be $\mathrm{Pi} \mathrm{Z}$ and $\mathrm{Pi} \mathrm{M}$ homozygotes respectively with measurable amounts of $\alpha_{1}$-AT, unlike null homozygotes where no $\alpha_{1}$-AT is present. Clues may arise if the offspring of a parent assumed to be a Pi Z homozygote appear phenotypically to be Pi M homozygote especially if the $\alpha_{1}$-AT concentration is reduced ( $\sim 50 \%$ normal).

The ELUCIGENE AAT kit detects the $\mathrm{S}$ allele, the $\mathrm{Z}$ allele and the normal sequences at the $S$ and $Z$ loci. This means that it can distinguish between heterozygotes and homozygotes for each allele as well as the compound heterozygote SZ. This study confirms that unusual variants (non-M) especially and most importantly the null variants can be identified by comparing the results with the concentration and phenotype. The $Z$ null heterozygotes appear, on the ELUCIGENE AAT to be similar to an MZ heterozygote as only one $\mathrm{Z}$ allele is detected. Assessment of the plasma would separate these possibilities as the $Z$ null variants have a concentration well below the normal range $(<10 \%)$ whereas the $\mathrm{MZ}$ heterozygote has a concentration close to the normal range $(\sim 60 \%)$. The same will also be true for the poorly functional phenotypes like FZ, where the genotype as determined by the current test would only detect $1 \mathrm{Z}$ allele and the plasma concentration would be similar to that of the more common MZ heterozygote.

Clearly the current methodology has to be interpreted with caution in the heterozygonous state as emphasized by the manufacturer. However, this methodology is readily adaptable to all potential phenotypes and could become a powerful epidemiological and diagnostic tool that would overcome the complex problems involved in the preparation and interpretation of phenotype gels. However, the cost of the commercial test (which has now become unavailable) has been GBP23 per sample which may prohibit wide scale usage for patient screening. Nevertheless this approach may eventually supercede phenotyping in selected patients for accurate diagnosis.

Acknowledgements. The authors acknowledge the technical assistance of D. Bayley, S. Howes, R. Ferrie and R. Lewis for typing the manuscript.

\section{References}

1. Bruce RM, Cohen BH, Diamond EL, et al. Collaborative study to assess risk of lung disease in Pi MZ phenotype subjects. Am Rev Respir Dis 1984; 130: $386-390$.

2. Lieberman J, Winter B, Statre A. Alpha ${ }_{1}$-antitrypsin Pi types in 965 COPD patients. Chest 1986; 89: $370-$ 373.

3. Turino GM, Barker AF, Brantly ML, et al. Clinical features of individual Pi SZ phenotype of alpha-1antitrypsin deficiency. Alpha-1-antitrypsin deficiency registry study group. Am J Respir Crit Care Med 1996; 154: $1718-1725$.

4. Cooke L, Burdon JDW, Brenton S, Knight KR, Janus ED. Kinetic characterisation of $\alpha_{1}$ antitrypsin $F$ as an inhibitor of neutrophil elastase. Pathol 1996; 28: 242 247.

5. Main BF, Jones PJ, MacGillivray RT, Banfield DK. Apolipoprotein E genotyping using the polymerase chain reaction and allele-specific oligonucleotide primers. J Lipid Res 1991; 32: 183-187.

6. Newton CR, Graham A, Heptinstall LE, et al. Analysis of any point mutation in DNA. The amplification refractory mutation system (ARMS). Nucleic Acids Res 1989; 17: 2503-2516.

7. Stoller JK, Smith P, Jang P, Spray J. Physical and social impact of alpha-1-antitrypsin deficiency: results of a male survey of the readership of a national newsletter. Cleve Clin J Med 1995; 61: 461-467.

8. Thelin T, Sveger T, McNeil TF. Primary prevention in high-risk group: smoking habits in adolescents with homozygous alpha-1-antitrypsin deficiency (ATD). Acta Paediatric 1996; 85: $1207-1212$.

9. Baur X, Bencze K. Study of familial alpha-1proteinase inhibitor deficiency including a rare proteinase inhibitor phenotype (IZ). Respiration 1987; 51: $188-195$.

10. Brantly M. Alpha-1-antitrypsin genotypes and phenotypes. In: Crystal RG, ed. Alpha-1-antitrypsin deficiency: Biology Pathogenesis Clinical manifestations Therapy. NY: Marcel Dekker Inc. 1996; pp. $211-226$. 